\title{
Factores asociados a hemoptisis en pacientes con tuberculosis pulmonar en el Hospital Nacional Dos de Mayo, Lima - Perú
}

\author{
Associated factors with hemoptys in patients with pulmonary tuberculosis at Dos de \\ Mayo National Hospital, Lima - Perú
}

\author{
Briam Benito Condor ${ }^{a}$, Carlos Contreras Camarena ${ }^{1, b}$, Rosa Justo Calle ${ }^{c}$, Felix Llanos Tejada ${ }^{2, d}$, \\ Juan Salas López ${ }^{2, e}$, Hector Jave Castillo ${ }^{2, f}$ \\ ${ }^{1}$ Departamento de Medicina, Hospital Nacional Dos de Mayo. Lima, Perú. \\ ${ }^{2}$ Servicio de Neumología, Hospital Nacional Dos de Mayo. Lima, Perú. \\ ${ }^{a}$ Médico cirujano, ORCID: https://orcid.org/0000-0003-3163-237X \\ ${ }^{\mathrm{b}}$ Médico internista, ORCID: https://orcid.org/0000-0002-7394-995X \\ cMédica cirujano, ORCID: https://orcid.org/0000-0001-7189-5575 \\ ${ }^{\mathrm{d}}$ Médico neumólogo, ORCID: https://orcid.org/0000-0003-1834-1287 \\ ${ }^{e}$ Médico neumólogo, ORCID: https://orcid.org/0000-0003-3686-6837 \\ 'Médico neumólogo, ORCID: https://orcid.org/0000-0001-9260-4298
}

An Fac med. 2020;81(4):398-403. / DOI: https://doi.org/10.15381/anales.v81i4.18748

Correspondencia:

Briam Benito Condor

briam.benito@gmail.com

Recibido: 7 de septiembre 2020

Aprobado: 19 de marzo 2021

Publicación en línea: 15 de abril 2021

Conflictos de interés: Los autores declaran no tener conflictos de interés.

Fuente de financiamiento: Autofinanciado

Citar como: Benito B, Contreras C, Justo $R$, Llanos F, Salas J, Jave H. Factores asociados a hemoptisis en pacientes con tuberculosis pulonar en el Hospital Nacional Dos de Mayo, Lima - Perú. An Fac med. 2020;81 (4):398-403. DOI: https://doi.org/10.15381/anales. v81i4.18748

\section{Resumen}

Objetivos. Determinar los factores asociados a hemoptisis en pacientes con tuberculosis pulmonar admitidos al Hospital Nacional Dos de Mayo. Métodos. Estudio analítico de casos y controles. Se seleccionaron pacientes con baciloscopia positiva del programa de tuberculosis del Hospital Nacional Dos de Mayo, Lima - Perú. Los casos fueron pacientes con tuberculosis pulmonar con hemoptisis; y los controles, tuberculosis pulmonar sin hemoptisis. Se estudiaron variables epidemiológicas, clínicas y laboratoriales. Se empleo el software SPSS 24. Se determinaron medidas de frecuencia, medidas de tendencia central y de dispersión, así como los odds ratio con sus respectivos intervalos de confianza al 95\%, se consideró estadísticamente significativo el valor de $p<0,05$. Resultados. La hemoptisis representó el $5,8 \%$ del total de pacientes con tuberculosis pulmonar y baciloscopia positiva, el $80 \%$ se presentó en varones menores de 60 años. El $70 \%$ de casos tuvo antecedente de hábito alcohólico (OR: 2,5 IC: 1,2-5,5); el $24 \%$ exposición a quema de biomasa (OR: 4,9 IC: 1,7-14); el 66\% presentó dolor torácico (OR: 3,3 IC: 1,6-6,7); las alteraciones tomográficas estuvieron presentes en el $88 \%$ de casos (OR: 24,5 IC: 9,2-64,8); no se encontró variaciones en el perfil de coagulación. La mortalidad por hemoptisis en pacientes hospitalizados con tuberculosis pulmonar con baciloscopía positiva fue del 20,22\%. Conclusiones. El alcoholismo crónico y la evidencia tomográfica de bronquiectasia estuvieron asociados a hemoptisis en pacientes hospitalizados por tuberculosis pulmonar con baciloscopia positiva.

Palabras clave: Hemoptisis; Tuberculosis Pulmonar; Factores de Riesgo; Alcoholismo; Perú (fuente: DeCS BIREME).

\section{Abstract}

Objectives. To determine the factors associated with hemoptysis in patients with pulmonary tuberculosis admitted at Dos de Mayo National Hospital. Methods. Analytical study of cases and controls. Patients from the tuberculosis program at Dos de Mayo National Hospital, Lima- Peru, were selected. The cases were patients with pulmonary tuberculosis with hemoptysis, and controls pulmonary tuberculosis without hemoptysis. Epidemiological, clinical and laboratory variables were studied. SPSS 24 software is used. Frequency, central trend and dispersion measurements were determined, as well as odds ratios with their respective confidence intervals at $95 \%$, the value of $p<0,05$ was considered statistically significant. Results. Tuberculosis hemoptysis account for $5,8 \%$ of all patients with pulmonary tuberculosis, $80 \%$ occurred in males under 60 years old. $70 \%$ of cases had a history of alcoholic habits (OR: $2,5 \mathrm{Cl}: 1,2-5,5$ ); $24 \%$ exposure to biomass burning (OR: 4,9 Cl: 1,7-14); $66 \%$ had chest pain (OR: 3,3 IC: 1,6-6,7); tomographic alterations were present in $88 \%$ of cases (OR: $24,5 \mathrm{IC}: 9,2-64,8)$; however, no variations in the coagulation profile were found. Death from hemoptysis in hospitalized patients with pulmonary tuberculosis was $20,22 \%$. Conclusions. Chronic alcoholism and tomographic evidence of bronchiectasis were associated with hemoptysis in patients hospitalized for pulmonary tuberculosis with positive smear microscopy.

Keywords: Hemoptysis; Tuberculosis, Pulmonary; Risk Factors; Alcoholism; Peru (source: MeSH NLM). 


\section{INTRODUCCIÓN}

Hemoptisis se define como la expulsión de sangre procedente del parénquima pulmonar mediante la tos en presencia de enfermedad pulmonar; de acuerdo con su severidad puede amenazar la vida del paciente. La tuberculosis (TB) es una enfermedad endémica en el Perú, que usualmente se asocia a hemoptisis. En una investigación realizada en el Hospital Nacional Dos de Mayo se encontró que más del $70 \%$ de las hemoptisis correspondieron a tuberculosis pulmonar y bronquiectasia ${ }^{(1)}$, otras investigaciones han reportado resultados distintos; asumiendo que los resultados pueden variar, según las características y la distribución geográfica de la población, con predominancia de ciertas patologías benignas o malignas ${ }^{(2,3)}$. Por otro lado, existen factores que condicionan o precipitan el sangrado broncopulmonar, tales como el alcohol, tabaquismo, quema de biomasa, exposición a tóxicos ambientales y presencia de comorbilidad (4,5,6); así, el abordaje de estas condiciones sería de gran utilidad para el manejo y control de la hemoptisis. Se estima que entre $5 \%$ y $14 \%$ de los pacientes con hemoptisis son cuadros severos y pueden comprometer la vida; en estos casos la mortalidad fluctúa entre $9 \%$ a $38 \%{ }^{(7)}$. La presencia de comorbilidad, y otros tóxicos pulmonares han sido variables estudiadas en pacientes en tratamiento con TB, con resultados negativos tanto en cumplimiento de tratamiento, recidiva y pronóstico final, con el consiguiente incremento de mortalidad. Asimismo, Bhalla y col. ${ }^{(8)}$ reportaron incremento considerable de mortalidad en presencia de sangrado broncopulmonar moderado a severo, especialmente aquellos pacientes con enfermedad pulmonar obstructiva (EPOC) y cáncer pulmonar. La infección crónica causada por el bacilo tuberculoso produce erosiones bronco vasculares, generando dilatación anatómica secuelar irreversible del circuito pulmonar conocidas como bronquiectasias. La erosión, la congestión vascular y las anastomosis entre la circulación pulmonar y bronquial son algunos de los mecanismos fisiopatológicos que ocurren en la enfermedad tuberculosa.

La mortalidad de hemoptisis por tuberculosis en el Hospital Nacional Dos de Mayo (HN2DM) se ha incrementado no- tablemente en estas últimas décadas, en el 2000 la mortalidad fue de $8 \%$, el 2012 $7,6 \%$ y en el 2016 la mortalidad ascendió a $16,48 \%{ }^{(9)}$. Este aumento fue atribuido por los investigadores al incremento de la incidencia de tuberculosis pulmonar multidrogoresistente, mayor comorbilidad, asociación frecuente con HIV-TB, drogadicción, alcoholismo, incremento de micosis en adultos jóvenes y elevada frecuencia de cáncer pulmonar; sin embargo, no solo se incrementa la mortalidad por hemoptisis, sino que, además, se incrementa el uso hospitalario de las camas clínicas, pues, los pacientes requieren ser hospitalizados con mayor frecuencia, especialmente los pacientes con grados moderados o severos de hemoptisis, o con algún deterioro hemodinámico que pone en riesgo la vida del paciente, requiriendo medidas que incluyen manejo de la vía aérea, reposición de volumen sanguíneo y con relativa frecuencia broncofibroscopía de emergencia.

El objetivo principal de la investigación fue determinar los factores asociados a hemoptisis en pacientes diagnosticados de tuberculosis pulmonar; los resultados serán relevantes para mejorar e implementar estrategias de prevención de hemoptisis, mejorar calidad de vida de los pacientes, disminuir morbimortalidad y la frecuencia de hospitalización de pacientes con hemoptisis por tuberculosis pulmonar.

\section{MÉTODOS}

\section{Diseño del estudio}

Se realizó un estudio observacional, analítico, de casos y controles. Los casos fueron los pacientes con TB pulmonar hospitalizados con hemoptisis y baciloscopia positiva. Los controles fueron los pacientes con TB pulmonar hospitalizados sin hemoptisis y baciloscopia positiva, los cuales se seleccionaron por aleatorización simple de la data del programa de control de tuberculosis (PCT) del HN2DM. La relación caso/control fue 1:2.

\section{Población de estudio}

La población fueron pacientes con diagnóstico de TB pulmonar hospitalizados por hemoptisis y registrados en el PCT, durante el periodo enero 2017 a diciembre 2019 en el HN2DM. La fuente de información fue la historia clínica, respetando su confidencialidad.

\section{Variables de estudio}

Se recolectaron variables sociodemográficas que incluyeron edad, sexo, nivel educativo, hábitos nocivos (alcohol, tabaco), exposición a tóxicos inhalantes (ocupacional, soldadura, pintura industrial) y a biomasa (quema de leña, combustión biológica). Se recolectaron variables clínicas como tiempo de enfermedad, sintomatología del paciente a su ingreso hospitalario y presencia de comorbilidad. Adicionalmente se registraron hallazgos radiológicos y tomográficos; finalmente, variables analíticas como la razón normalizada internacional (INR), tiempo de protrombina (TP), tiempo de tromboplastina parcial (TTP), recuento plaquetario, farmacorresistencia del bacilo tuberculoso: TB sensible, MRD o XDR.

\section{Análisis estadístico}

Los datos recolectados fueron codificados y procesados con el programa SPSS, versión 24.0. Los resultados se expresaron en frecuencias absolutas y relativas para variables cualitativas. La media y la desviación estándar fueron las medidas usadas en el procesamiento de variables cuantitativas. Se elaboraron tablas de contingencia de $2 \times 2$ dentro del análisis bivariado. En el caso de las variables dicotómicas se determinó el odds ratio con su respectivo intervalo de confianza al 95\%, se consideró $p<$ 0,05 como estadísticamente significativo. Se utilizó el modelo de regresión logística para la determinación de factores asociados. Finalmente se analizó el sesgo de la edad y el genero en el grupo control usando $T$ de student para muestras independientes y chi cuadrado respectivamente.

\section{Aspectos éticos}

La investigación tuvo la aprobación del Comité de Ética del Hospital Nacional Dos de Mayo. Debido a que se usó la historia clínica como fuente de información, no se hizo uso del consentimiento informado; sin embargo, se mantuvo la confidencialidad de los datos.

\section{RESULTADOS}

Entre enero de 2017 a diciembre 2019 se encontraron 1218 pacientes diagnosti- 
cados de TB pulmonar confirmados bacteriológicamente y registrados en el PCT durante el periodo de estudio. Se hospitalizaron 89 pacientes con diagnóstico de hemoptisis al ingreso, de los cuales fallecieron 18, quedando 71 pacientes con hemoptisis que requirieron hospitalización (hemoptisis moderada o masiva). Se descartaron los pacientes fallecidos y 21 casos por información incompleta. La mortalidad por hemoptisis en pacientes diagnosticados de TB pulmonar fue de $20,22 \%$.

El resultado del análisis de las características generales de los casos y de los controles no identificó diferencia significativa en cuanto a la edad, sexo y nivel educativo; de tal manera que se pueden realizar comparaciones entre ambos grupos (Tabla 1).

El promedio de edad fue 43,88 \pm 20,66 años en el grupo de casos y 41,8 $\pm 19,61$ en los controles; el $44 \%$ y el $48 \%$ tuvieron menos de 40 años en el grupo de casos y control, respectivamente. El género masculino predominó en ambos grupos, $80 \%$ y $69 \%$, respectivamente, sin diferencias significativas entre ambos grupos ( $p<0,05)$. 11 pacientes ( 3 con diabetes mellitus, 3 con asma bronquial, 3 con neoplasias malignas y 2 con HIV), tenían comorbilidad en el grupo de casos y 41 pacientes (12 con diabetes mellitus, 18 con HIV, 2 con asma bronquial, 9 con otras patologías), en el grupo control. El antecedente de alcoholismo crónico se encontró presente en el 70 \% de casos y en el $48 \%$ de los controles [OR: 2.5 (IC $95 \%$ : 1,2 - 5,1), p: 0,01]. El tabaquismo, fue registrado en el $24 \%$ y $20 \%$ de casos y controles respectivamente. El 30\% de casos estuvieron expuestos a tóxicos inhalantes, mientras que la exposición en el grupo control fue de 15\% [OR: 2.4 (IC 95\%: 1,07-5,4), p: 0,03]. En el 24\% de casos y en el $6 \%$ de controles registraron tener exposición a biomasa [OR: 4,9 (IC 95 \%: 1,7 - 14,1), p: 0,001]. Las variables edad, género y tabaquismo, no resultaron ser variables asociadas a hemoptisis. Tabla 2.

Los pacientes que presentaron dolor torácico, tuvieron tres veces más probabilidad de presentar hemoptisis moderada a severa [OR: 3,3 (IC95 \%: 1,6-6,7)], comparados con el grupo control. No se encontraron diferencias estadísticamente significativas en el análisis bivariado respecto a variables clínicas como tiempo de enfermedad, pérdida de peso, anorexia, diaforesis, sensación de alza térmica, disnea y comorbilidad.

Respecto a los hallazgos radiológicos y tomográficos, la presencia de bronquiectasia fue considerablemente mayor en el grupo de casos comparados con el grupo control, (52 \% vs 6\%). La cavitación fue más frecuente en los casos (44\%), comparados con el grupo control (9\%); ambas alteraciones pulmonares tuvieron asociación estadísticamente significativa con hemoptisis. La localización cavitaria más frecuente en el grupo de casos fue la apical, mientras que

Tabla 1. Caracteristicas generales de los paciententes con tuberculosis pulmonar hospitalizados por hemoptisis (casos) y sin hemoptisis (controles), en el Hospital Nacional Dos de Mayo, 2017-2019.

\begin{tabular}{|c|c|c|c|c|c|c|c|c|}
\hline & & \multicolumn{4}{|c|}{ Grupo de estudio } & \multirow{2}{*}{\multicolumn{2}{|c|}{ Total }} & \multirow{3}{*}{$\mathbf{p}$} \\
\hline & & \multicolumn{2}{|c|}{ Controles } & \multicolumn{2}{|c|}{ Casos } & & & \\
\hline & & $n$ & $\%$ & $\mathbf{n}$ & $\%$ & n & $\%$ & \\
\hline \multirow{2}{*}{ Edad } & $=<40$ años & 56 & $56.0 \%$ & 26 & $52.0 \%$ & 82 & $54.7 \%$ & \multirow{2}{*}{0,63} \\
\hline & >40 años & 44 & $44.0 \%$ & 24 & $48.0 \%$ & 68 & $45.3 \%$ & \\
\hline \multirow{2}{*}{ Género } & Varón & 40 & $80,0 \%$ & 69 & $69,0 \%$ & 109 & $72,7 \%$ & \multirow{2}{*}{0,15} \\
\hline & Mujer & 10 & $20,0 \%$ & 31 & $31,0 \%$ & 41 & $27,3 \%$ & \\
\hline \multirow{3}{*}{ Nivel educativo } & Sin educación & 8 & $8.0 \%$ & 1 & $2.0 \%$ & 9 & $6.0 \%$ & \multirow{3}{*}{0,05} \\
\hline & Básica & 70 & $70.0 \%$ & 44 & $88.0 \%$ & 114 & $76.0 \%$ & \\
\hline & $\begin{array}{c}\text { Técnico- } \\
\text { universitario }\end{array}$ & 22 & $22.0 \%$ & 5 & $10.0 \%$ & 27 & $18.0 \%$ & \\
\hline
\end{tabular}

$* p<0,05$ significativo la localización parahiliar fue la localización más frecuente en el grupo control. Tabla 3.

No se encontraron diferencias estadísticamente significativas respecto a variables analíticas como perfil de coagulación, recuento plaquetario y hemoglobina. La media de la hemoglobina se encontró por debajo de lo normal en ambos grupos, $11,18 \pm 1,80$ en el grupo de casos y 11,23 $\pm 2,08$ (p: 0,09) en el grupo control; el perfil de coagulación (INR, TP, TTPA) y el recuento plaquetario se mantuvieron dentro del rango normal sin diferencia estadística.

Utilizando las variables que resultaron estadísticamente significativas producto del análisis bivariado, se procedió a ingresar las variables significativas $(p<0,05)$, al modelo de regresión logística múltiple, según se muestra en la tabla 4. En el paso 11 del modelo predictivo se encontró que la presencia alcoholismo crónico incrementó en 3 veces más la probabilidad de aparición de hemoptisis. Los pacientes con bronquiectasia tuvieron 5 veces más probabilidad de presentar hemoptisis. Paradójicamente, los pacientes con infección por el virus de la inmunodeficiencia humana (HIV) tuvieron una probabilidad disminuida o protectora para hemoptisis (OR: 0,03).

Finalmente se realizó el análisis del sesgo que representa las comorbilidades en el grupo control según edad y sexo. La edad no presentó diferencia significativa según la comorbilidad en el grupo control (Tabla 5). Del análisis del género se puede apreciar que no son independientes, siendo la misma proporción $p=0,23>0,05$ en el grupo control (Tabla 6). El análisis de sesgo permite validar los resultados del estudio.

\section{DISCUSIÓN}

La prevalencia de hemoptisis en pacientes hospitalizados y diagnosticados de TB con baciloscopia positiva fue 5,82\%. La mortalidad total encontrada en pacientes hospitalizados por hemoptisis moderada a severa fue $20,22 \%$, resultado similar a otros estudios, que atribuyen una mortalidad del $7 \%$ al $30 \%$ en la he- 
Tabla 2. Factores asociados a hemoptisis en pacientes con tuberculosis pulmonar. Hospital Nacional Dos de Mayo, 2017- 2019.

\begin{tabular}{|c|c|c|c|c|c|c|c|c|}
\hline \multirow{2}{*}{ Variables } & & \multicolumn{2}{|c|}{ Casos } & \multicolumn{2}{|c|}{ Controles } & \multirow{2}{*}{ OR } & \multirow{2}{*}{ IC $95 \%$} & \multirow{2}{*}{$\mathbf{p}$} \\
\hline & & $n$ & $(\%)$ & $n$ & (\%) & & & \\
\hline \multirow{2}{*}{ Edad } & > 40 años & 24 & 48 & 44 & 44 & \multirow{2}{*}{1,17} & \multirow{2}{*}{$<0,59-2,32>$} & \multirow{2}{*}{0,64} \\
\hline & $\leq 40$ años & 26 & 52 & 56 & 56 & & & \\
\hline \multirow{2}{*}{ Género } & Varón & 40 & 80 & 69 & 69 & \multirow{2}{*}{1,79} & \multirow{2}{*}{$<0,7-4>$} & \multirow{2}{*}{0,15} \\
\hline & Mujer & 10 & 20 & 31 & 31 & & & \\
\hline \multirow{2}{*}{ Alcohol } & Sí & 35 & 70 & 48 & 48 & \multirow{2}{*}{2,5} & \multirow{2}{*}{$<1,2-5,1>$} & \multirow{2}{*}{$0,01^{*}$} \\
\hline & No & 15 & 30 & 52 & 52 & & & \\
\hline \multirow{2}{*}{ Tabaquismo } & Sí & 12 & 24 & 20 & 20 & \multirow{2}{*}{1,3} & \multirow{2}{*}{$<0,5-2,8>$} & \multirow{2}{*}{0,57} \\
\hline & No & 38 & 76 & 80 & 80 & & & \\
\hline \multirow{2}{*}{$\begin{array}{l}\text { Exposición } \\
\text { tóxicos } \\
\text { inhalantes }\end{array}$} & Sí & 15 & 30 & 15 & 15 & \multirow{2}{*}{2,4} & \multirow{2}{*}{$<1,07-5,4>$} & \multirow{2}{*}{$0,03^{*}$} \\
\hline & No & 35 & 70 & 85 & 85 & & & \\
\hline \multirow{2}{*}{$\begin{array}{l}\text { Exposición } \\
\text { biomasa }\end{array}$} & Sí & 12 & 24 & 6 & 6 & \multirow{2}{*}{4,9} & \multirow{2}{*}{$<1,7-14,1>$} & \multirow{2}{*}{$0,001^{*}$} \\
\hline & No & 38 & 76 & 94 & 94 & & & \\
\hline Total & & 50 & 100 & 100 & 100 & & & \\
\hline
\end{tabular}

${ }^{*} p<0,05$ significativo

moptisis no masiva y de $75 \%$ en la hemoptisis masiva ${ }^{(9,10)}$.

Los pacientes con hábitos nocivos, específicamente alcoholismo crónico, tuvieron 3 veces más riesgo de hemoptisis, probablemente relacionado con abandono terapéutico, malnutrición y altera-

Tabla 3. Riesgo de hemoptisis en pacientes con tuberculosis pulmonar, según hallazgos radiológicos. Hospital Nacional Dos de Mayo, 2017- 2019.

\begin{tabular}{|c|c|c|c|c|c|c|c|c|}
\hline & & \multicolumn{2}{|c|}{ Casos } & \multicolumn{2}{|c|}{ Controles } & \multirow[t]{2}{*}{ OR } & \multirow[t]{2}{*}{ IC 95\% } & \multirow[t]{2}{*}{$\mathbf{P}$} \\
\hline & & $\mathbf{n}$ & (\%) & $\mathbf{n}$ & (\%) & & & \\
\hline \multirow{2}{*}{$\begin{array}{l}\text { Anomalías } \\
\text { radiológicas }\end{array}$} & Sí & 44 & 88 & 23 & 23 & & & \\
\hline & No & 6 & 12 & 77 & 77 & 24,5 & $\langle 9,2-64,8\rangle$ & $0,000 *$ \\
\hline \multirow{2}{*}{ Bronquiectasias } & Sí & 26 & 52 & 6 & 6 & & & \\
\hline & No & 24 & 48 & 94 & 94 & 16,9 & $<6,2-45,8>$ & $0,000^{*}$ \\
\hline \multirow{2}{*}{ Cavitación } & Sí & 22 & 44 & 9 & 9 & & & \\
\hline & No & 28 & 56 & 91 & 91 & 7,9 & $<3,2-19,2\rangle$ & $0,000 *$ \\
\hline \multirow{2}{*}{ Consolidación } & Sí & 6 & 12 & 10 & 10 & & & \\
\hline & No & 44 & 88 & 90 & 90 & 1,2 & $<0,4-3,5>$ & 0,70 \\
\hline \multirow{3}{*}{$\begin{array}{l}\text { Localización de } \\
\text { caverna }\end{array}$} & Apical & 17 & 77,3 & 1 & 11,1 & & & \\
\hline & Hiliar & 2 & 9,1 & 3 & 33,3 & & & $0,02 *$ \\
\hline & Parahiliar & 3 & 13,6 & 5 & 55,6 & & & \\
\hline
\end{tabular}

${ }^{*} p<0,05$ significativo las células inmunes como el macrófago alveolar y neutrófilos, reduciendo las defensas de la barrera epitelial en las vías aéreas bajas, haciéndolo susceptible al daño y/o colonización de patógenos ${ }^{(4,5)}$.

Los pacientes con exposición a inhalantes químicos (derivados de pinturas laca, de soldadura eléctrica y autógena) tuvieron 2,4 veces más riesgo de presentar hemoptisis que los no expuestos. Estos hallazgos sugieren asociación entre agentes tóxicos inhalantes pulmonares y el desarrollo de hemoptisis. Los pacientes con tuberculosis pulmonar expuestos a combustión de biomasa (humo originado de cualquier proceso biológico derivado de vegetales, animales y sus derivados), tuvieron 4,9 veces más probabilidad de presentar hemoptisis, posiblemente relacionado al efecto inflamatorio crónico del humo, infiltración neutrofilica, desactivación del surfactante pulmonar y expresión de metaloproteinasas que este produce, promoviendo y desencadenando la aparición de asma, tuberculosis, EPOC y otras enfermedades pulmonares crónicas ${ }^{(11)}$. Es decir, la exposición a biomasa llevaría a un estado proinflamatorio sistémico, modificando las características clínicas y el pronóstico de la TB. El metaanálisis realizado por Shegal y col. (12) reportó entre 2 a 3 veces más la probabilidad de presentar TB en personas con exposición a biomasa, especialmente aquellos que cocinaban con leña por más de 10 años.

El estudio determinó que los pacientes que presentaron tos productiva y dolor torácico tuvieron 4,2 y 3,3 veces más riesgo de hemoptisis respectivamente. Estos hallazgos probablemente están relacionados con la localización periférica de las lesiones, cercanía de la pleura y con aumento de la presión intratorácica con la tos. Estos resultados concuerdan con los encontrados por Earwood y col. ${ }^{(13)}$ y Wong y col. ${ }^{(14)}$ quienes encontraron asociación entre hemoptisis, tos productiva y dolor torácico.

El 22\% de casos registraron tener algún tipo comorbilidad en comparación con el $41 \%$ de pacientes del grupo control. La proporción elevada de comorbilidad en el grupo control probablemente se produjo por el elevado número de pacientes con HIV que fueron admitidos a 
Tabla 4. Factores asociados a hemoptisis en tuberculosis pulmonar Hospital Nacional Dos de Mayo, 2017- 2019. Análisis multivariado de regresión logística.

\begin{tabular}{|c|c|c|c|c|}
\hline \multirow{2}{*}{ Variables } & \multirow{2}{*}{ Significancia } & \multirow{2}{*}{$\operatorname{Exp}(B)$} & \multicolumn{2}{|c|}{ IC $95 \%$} \\
\hline & & & Inferior & Superior \\
\hline Alcoholismo & 0,033 & 3,23 & 1,102 & 9,505 \\
\hline Anomalías radiológicas & 0,000 & 15,91 & 4,797 & 52,789 \\
\hline Bronquiectasia & 0,009 & 5,85 & 1,562 & 21,973 \\
\hline VIH & 0,005 & 0,03 & 0,003 & 0,341 \\
\hline Constante & 0,999 & 0,00 & & \\
\hline
\end{tabular}

Tabla 5. Análisis de sesgo de pacientes con hemoptisis por tuberculosis pulmonar, con comorbilidades, según edad en el grupo control. Hospital Nacional Dos de Mayo, 2017- 2019.

\begin{tabular}{ccccccc} 
& & $\mathbf{n}$ & Media & $\begin{array}{c}\text { Desviacion } \\
\text { estándar }\end{array}$ & T & p \\
Comorbilidad & No & 59 & 39,8 & 21,3 & 2,8 & 0,22 \\
\hline
\end{tabular}

T student para muestras independientes $p=0,22>0,05$

Tabla 6. Análisis de sesgo de pacientes con hemoptisis por tuberculosis pulmonar, con comorbilidades, según sexo en el grupo control. Hospital Nacional Dos de Mayo, 2017- 2019.

\begin{tabular}{|c|c|c|c|c|c|c|c|}
\hline & & \multicolumn{4}{|c|}{ Comorbilidad } & \multirow{2}{*}{\multicolumn{2}{|c|}{ Total }} \\
\hline & & \multicolumn{2}{|c|}{ No } & \multicolumn{2}{|c|}{ Sí } & & \\
\hline & & $n$ & $\%$ & $n$ & $\%$ & $\mathbf{n}$ & $\%$ \\
\hline \multirow[t]{3}{*}{ Género } & Varón & 38 & $64,4 \%$ & 31 & $75,6 \%$ & 69 & $69 \%$ \\
\hline & Mujer & 21 & $35,6 \%$ & 10 & $24,4 \%$ & 31 & $31 \%$ \\
\hline & Total & 59 & $100 \%$ & 41 & $100 \%$ & 100 & $100 \%$ \\
\hline
\end{tabular}

Chi cuadrado: $1,21 \mathrm{p}=0,23>0,05$

las salas de medicina, neumología e infectología, posiblemente por su condición de HIV - SIDA que por la propia hemoptisis. Es conocido que los pacientes con coinfección de HIV-TB, presentan clínica menos florida que el paciente inmunocompetente, por lo tanto, es menos probable encontrar tos crónica productiva y hemoptisis, lo que puede obedecer a su menor capacidad para formar cavitaciones pulmonares, escaza necrosis tisular y menor inflamación alveolo bronquial (15). Los datos indican que los pacientes con comorbilidad específicamente por HIV tienen 20\% menos riesgo de hemoptisis; este hallazgo probablemente se explique por la inmunodepresión que incrementaría la replicación y el desarrollo de la enfermedad, pero produciría un enmascaramiento de los síntomas graves; el mejor ejemplo es el empeoramiento clínico pa- radójico tras la reconstitución inmune del tratamiento antirretroviral ${ }^{(16,17,18)}$.

En nuestra serie, el $88 \%$ de casos y $23 \%$ de controles tuvieron anomalías radiológicas o tomográficas; según el análisis de regresión logística, estos pacientes tuvieron alrededor de 15 veces mayor riesgo de presentar hemoptisis, siendo mayor la probabilidad de sangrado en las bronquiectasias (OR: 16,9 IC: 6,2 - 45,8) y cavitaciones (OR: 7,9 IC: 3,2 - 19,2), resultado similar a lo reportado por Tan y Parmasivan quienes encontraron una mayor proporción de hemoptisis en pacientes con lesiones cavitarias ${ }^{(19,20)}$.

No se encontraron diferencias significativas en relación con el perfil laboratorial bioquímico, hematológico y de coagulación, hallazgo congruente con Soares y col. ${ }^{(21)}$ quien encontró que el $70 \%$ de pacientes con hemoptisis no tuvo alteración en el perfil de coagulación.

Las limitaciones del estudio fueron la naturaleza retrospectiva de la investigación y el tamaño reducido de la población, por lo que es necesario diseñar estudios longitudinales y con mayor población para corroborar estos resultados. La muestra hospitalaria puede ser extrapolada solo a pacientes hospitalizados con diagnóstico de TB y hemoptisis, mas no a pacientes de atención primaria o ambulatoria. En los adultos mayores con tabaquismo y hemoptisis no se hizo el estudio de descarte de neoplasia pulmonar, importante causa de hemoptisis en nuestro hospital.

Concluimos que para la población de estudio utilizada, los factores asociados a hemoptisis en pacientes hospitalizados por TB pulmonar fueron: alcoholismo crónico y evidencia tomográfica de bronquiectasia. No se encontró asociación estadísticamente significativa entre perfil de coagulación, valor de hemoglobina, drogo resistencia y otras variables sociodemográficas.

\section{REFERENCIAS BIBLIOGRÁFICAS}

1. Contreras C, Castro R, Pascacio M, Jave O, Llanos F. Causas de hemoptisis en pacientes hospitalizados. Rev Soc Peru Med Interna. 2013; 26 (3):11015. DOI: https://doi.org/10.36393/spmi.v26i3.403

2. Fidan A, Ozdoğan S, Oruç O, Salepçi B, Ocal Z, Cağlayan B. Hemoptysis: a retrospective analysis of 108 cases. Respir Med. 2002; Sep;96(9):677-80. DOI: 10.1053/rmed.2002.1359. PMID: 12243312.

3. Al-Nesf MA, Jerobin J, Al-Alawi AA, El-Kassim M, Mobayed $\mathrm{H}$, Mohammed TRN. Etiology and outcome of hemoptysis in Qatar, a high-resource country with a large expatriate population: A retrospective study. Qatar Med J. 2019; 1:1-9. DOI: https://doi. org/10.5339/qmj.2019.1

4. Lesnic E, Zlepca V. Impact of tobacco smoking on anti-tuberculosis treatment outcomes. Eur Respir J. 2013; 42(57):P4265.

5. Wessels J, Walsh CM, Nel M. Smoking habits and alcohol use of patients with tuberculosis at Standerton Tuberculosis Specialised Hospital, Mpumalanga, South Africa. Health SA. 2019 Oct 8;24:1146. DOI: 10.4102/hsag.v24i0.1146

6. Sarkar D, Jung MK, Wang HJ. Alcohol and the Immune System. Alcohol Res. 2015;37(2):153-5.

7. Kathuria H, Hollingsworth HM, Vilvendhan R, Reardon C. Management of life-threatening hemoptysis. J Intensive Care. 2020 Apr 5;8:23. DOI: 10.1186/ s40560-020-00441-8

8. Bhalla A, Pannu AK, Suri V. Etiology and outcome of moderate-to-massive hemoptysis: Experience from a tertiary care center of North India. Int J 
Mycobacteriol. 2017 Jul-Sep;6(3):307-310. DOI: 10.4103/ijmy.ijmy_54_17

9. Contreras C, Jave O, Cortez A, Lira H. Predictores de mortalidad en pacientes con hemoptisis en un servicio de emergencia. An Fac med. 2016;77(4):373-8. DOI: https://doi.org/10.15381/ anales.v77i4.12653

10. Radchenko C, Alraiyes AH, Shojaee S. A systematic approach to the management of massive hemoptysis. J Thorac Dis. 2017 Sep;9(10):S1069S1086. DOI: 10.21037/jtd.2017.06.41

11. Lopez M, Mongilardi N, Checkley W. Enfermedad pulmonar obstructiva crónica por exposición al humo de biomasa. Rev. perú. med. exp. salud publica. 2014; 31(1): 94-99.

12. Sehgal M, Rizwan SA, Krishnan A. Disease burden due to biomass cooking-fuel-related household air pollution among women in India. Glob Health Action. 2014;7:25326. DOI: 10.3402/gha.v7.25326
13. Earwood JS, Thompson TD. Hemoptysis: evaluation and management. Am Fam Physician. 2015; 91(4):243-9.

14. Wong CM, Lim KH, Liam CK. The causes of haemoptysis in malaysian patients aged over 60 and the diagnostic yield of different investigations. Respirology. 2003; 8(1):65-8. DOI: 10.1046/j.1440-1843.2003.00437.x

15. Pan American Health Organization. TB/HIV Coinfection Regional Clinical Manual. 2017 Update. Washington, D.C.: PAHO; 2018

16. Lawn SD, Wilkinson RJ, Lipman MC, Wood R. Immune reconstitution and "unmasking" of tuberculosis during antiretroviral therapy. Am J Respir Crit Care Med. 2008 Apr 1;177(7):680-5. DOI: 10.1164/ rccm.200709-1311PP

17. Manabe YC, Breen R, Perti T, Girardi E, Sterling TR. Unmasked tuberculosis and tuberculosis immune reconstitution inflammatory disease: a disease spectrum after initiation of antiretroviral therapy. J Infect Dis. 2009 Feb 1;199(3):437-44 DOI: 10.1086/595985

18. Wendel KA, Alwood KS, Gachuhi R, Chaisson RE, Bishai WR, Sterling TR. Paradoxical worsening of tuberculosis in HIV-infected persons. Chest. 2001 Jul;120(1):193-7. DOI: 10.1378/chest.120.1.193

19. Tan S, Sun D, Zhang T, Li Y, Cao Y, Njire M, et al. Risk Factors for Hemoptysis in Pulmonary TubercuIosis Patients from Southern China: A Retrospective Study. Journal of Tuberculosis Research. 2014; 2: 173-180. DOI: 10.4236/jtr.2014.24022

20. Paramasivan R, Rajasekaran S, Parthasarathy RT. Haemoptysis and pulmonary tuberculosis - An Analysis. Lung India. 1994, 12 (04): 192-194

21. Soares Pires F, Teixeira N, Coelho F, Damas C. Hemoptysis--etiology, evaluation and treatment in a university hospital. Rev Port Pneumol. 2011;17(1):714. DOI: 10.1016/s2173-5115(11)70004-5 